and Differentiation in the Cellular Slime Moulds (Eds Cappuccinelli, P., Ashworth, J.) 31, (Elsevier/North Holland, 1977)) it was suggested that $\mathrm{NH}_{3}$ inhibited spore and stalk cell differentiation by inhibiting cyclic AMP production. Recent work of Schindler and Sussman (Biochem. biophys. Res. Commun. 79, 611; 1977) showing that $\mathrm{NH}_{3}$ (but not $\mathrm{NH}_{4}{ }^{+}$) at physiological levels dramatically decreases cyclic AMP synthesis or release in starving cell suspensions and the work of Thadani, Pan and Bonner (Expl. Cell Res. 108, 75; 1977) showing an inhibitory effect of $\mathrm{NH}_{3}$ on cyclic AMP release by cells of the species $D$. mucoroides, suggest that this conjecture may indeed be soundly based. Whether the effect is indirect or whether $\mathrm{NH}_{3}$ acts directly on the membrane-bound adenylate cyclase remains to be resolved. $\square$

\section{Energetic oxygen ions in the magnetosphere}

from D. T. Young

MAGNETIC storms are vast disturbances of the Earth's magnetosphere dissipating energy at the rate of $10^{11}-10^{12}$ watts over several days. Their connection to disturbances on the Sun was realised more than 100 years ago and it is now clear that solar weather is carried to the Earth by the flow of solar wind plasma past the magnetosphere. Disturbances in the solar wind step up the vast magnetospheric dynamo thereby providing the storm's energy. But basically magnetic storms require the acceleration and transport of large amounts of hot plasma $(0.1 \sim 50 \mathrm{keV})$ and it is something of an embarrassment that no one really knows where all this plasma comes from. Does it originate as cold $(\lesssim 0.1 \mathrm{eV})$ ionospheric plasma or as relatively hot solar wind (electrons $\sim 10 \mathrm{eV}$, ions $\sim 1 \mathrm{keV}$ per nucleon)? Given what is known about magnetic storms and their relation to the solar wind the most reasonable assumption to make is that the hot plasma also originates in the solar wind. That this is not entirely the case has been demonstrated convincingly by the recent observation during three magnetic storms of roughly cqual amounts of hot $\mathrm{O}^{+}$ and $\mathrm{H}^{+}$ions $(0.5-16 \mathrm{keV})$ in the magnetospheric ring current (Johnson et al. Geophys. Res. Lett. 4, 403; 1977).

Centred about the equatorial plane, the ring current is an enormous electric

D. T. Young is at the Physikalisches Institut, University of Bern. circuit whose current is carried primarily by ions trapped in the geomagnetic field. Although the 'ring' occupies much the same region of space as the Van Allen radiation 'belits' the latter are much less dense and contribute very little net current. Magnetic storms are manifestations of the growth and decay of the ring current as hot plasma is injected into it early in the storm and is then gradually lost, primarily by charge exchange with neutral hydrogen and by scattering into the atmosphere. During storms the plasma Farticles penetrate deeper into the geomagnetic field and the quiescent ring current (normally centred at altitudes of $\approx 30,000 \mathrm{~km}$ ) shrinks down to altitudes of $\approx 10,000 \mathrm{~km}$. Although Johnson and his colleagues at the Lockheed Palo Alto Research Laboratory obtained data at somewhat lower altitudes $(\lesssim 8,000 \mathrm{~km})$, the bounce motion of the ions along magnetic lines of force enables them to sample the actual current carriers.

Together with the Lockheed group's earlier report of $\mathrm{O}^{+}$and $\mathrm{H}^{+}$ions streaming upwards out of the ionosphere (Shelley et al. Geophys. Res. Lett. 3, 654; 1976) their present results have far reaching consequences for models of the aurora and storm-time ring current. Most of these rely heavily on the hypothesis that solar wind plasma enters the magnetosphere by way of the magnetotail on the night side of the Earth. Plasma acceleration and transport deeper into the magnetosphere occurs duning the 'collapse' of part of the magnetotail and the accompanying conversion of stored magnetic energy into particle kinetic energy. As the heated plasma is driven deeper into the nightside magnetosphere, the more easily scattered electrons travel down magnetic field lines, bombarding the atmosphere and producing the aurora. Gradients in the geomagnetic field cause the relatively more stable ions to continue drifting in a westerly direction eventually forming the ring current. This explains why what was often called the 'proton' ring current was expected to have solar wind like composition and why it is so disconcerting to find terrestrial ions mixed in apparently large proportion. How the generally accepted hypothesis of plasma transport and acceleration from the magnetotail earthwards will be reconciled with the Lockheed group's observation of ionospheric plasma accelerated in more or less the opposite direction remains to be seen.

Johnson et al. obtained their data with one of a new class of particle detectors, the energetic ion mass spectrometer. Only in the past few years has it been technically feasible to build mass spectrometers with the required wide energy and mass ranges (typically $\approx 0-15 \mathrm{keV}$ per charge and 1 to $>30$ a.m.u. respectively) within the rigid constraints of low weight and stray magnetic fields demanded of satellite instrumentation. Perhaps the most sophisticated of the new spectrometers was first flown on the ESA GEOS satellite (Balsiger et al. Space Sci. Instrum. 2, 499; 1976) while the more recently launched ISEE-A carries a nearly identical spectrometer built by the Lockheed and GEOS groups together. To appreciate that these instruments are indeed a step forward one need only consider that before 1972 , plasma detectors operating between $10 \mathrm{eV}$ and $50 \mathrm{keV}$ were either electrostatic analysers or solid state devices used only to measure particle energy and classify their charge as positive or negative. Since solar wind plasma is known to be $\approx 95 \%$ protons the energyonly detectors abetted the hypothesis that the hot magnetospheric plasma originates in the solar wind. With this assumption to go on it was not necessary to measure anything but 'protons'.

Adding to the confusion over magnetospheric plasma origins brought on by the Lockheed results are observations made with the GEOS mass spectrometer of $\mathrm{O}^{2+}$ and ${ }^{4} \mathrm{He}^{2+}$ inferred to be of terrestrial origin (Young et al. Geophys. Res. Lett. 4, 561; 1977). We conclude that the ions are produced at altitudes $\gtrsim 20,000 \mathrm{~km}$ (that is, not the ionosphere) and are accelerated during storms to energies as high as $1 \mathrm{keV}$. This result is disconcerting because it was long thought that the simplest test for solar wind plasma in the magnetosphere would be the ratio of ${ }^{4} \mathrm{He}^{2+}$ to protons. This is about 0.04 in the solar wind and, before GEOS, the terrestrial value at $\mathrm{keV}$ energies was thought to be orders of magnitude smaller. The ${ }^{4} \mathrm{He}^{2+}$ ion is even further compromised as a tracer because it is converted rather quickly by charge exchange to ${ }^{4} \mathrm{He}^{+}$. As tracers one is apparently left only with more exotic (and difficult to measure) solar wind species such as $\mathrm{O}^{6+}$ and ${ }^{3} \mathrm{He}$. The latter has in fact been detected inside the magnetosphere by the foil collection technique.

Mass spectrometers have been something of an ugly duckling in magnetospheric research. Initially too heavy, magnetically dirty and ill suited for hot plasma measurements, they have come of age and are now invited to all the best satellite projects. The GEOS-ISEE type spectrometer will be flown on GEOS2 scheduled for launch this year, and is included on NASAs next magnetospheric research satellite the Dynamics Explorer. It therefore seems likely that energetic ion mass spectrometers will have the opportunity to supply a few answers to the many questions they have been raising lately. 